In Situ

Revue des patrimoines

$4 \mid 2004$

Les réseaux de la villégiature

\title{
Les réseaux de la villégiature en France
}

\section{Bernard Toulier}

\section{OpenEdition}

Journals

Édition électronique

URL : http://journals.openedition.org/insitu/1348

DOI : 10.4000/insitu. 1348

ISSN : 1630-7305

\section{Éditeur}

Ministère de la Culture

Référence électronique

Bernard Toulier, "Les réseaux de la villégiature en France », In Situ [En ligne], 4 | 2004, mis en ligne le 01 mars 2004, consulté le 21 septembre 2020. URL : http://journals.openedition.org/insitu/1348 DOI : https://doi.org/10.4000/insitu.1348

Ce document a été généré automatiquement le 21 septembre 2020

\section{(c) (i) $\odot$}

In Situ Revues des patrimoines est mis à disposition selon les termes de la licence Creative Commons Attribution - Pas d'Utilisation Commerciale - Pas de Modification 4.0 International. 


\section{Les réseaux de la villégiature en France}

\section{Bernard Toulier}

${ }^{1}$ La création des stations de villégiature, balnéaires, thermales ${ }^{2}$, climatiques ou des bords de ville s'inscrit à la fois dans le mouvement de l'avènement des loisirs et de la médicalisation de la société française du XIXe siècle, le développement des moyens de transport et l'accroissement exponentiel du tourisme ${ }^{3}$.

Figure 1

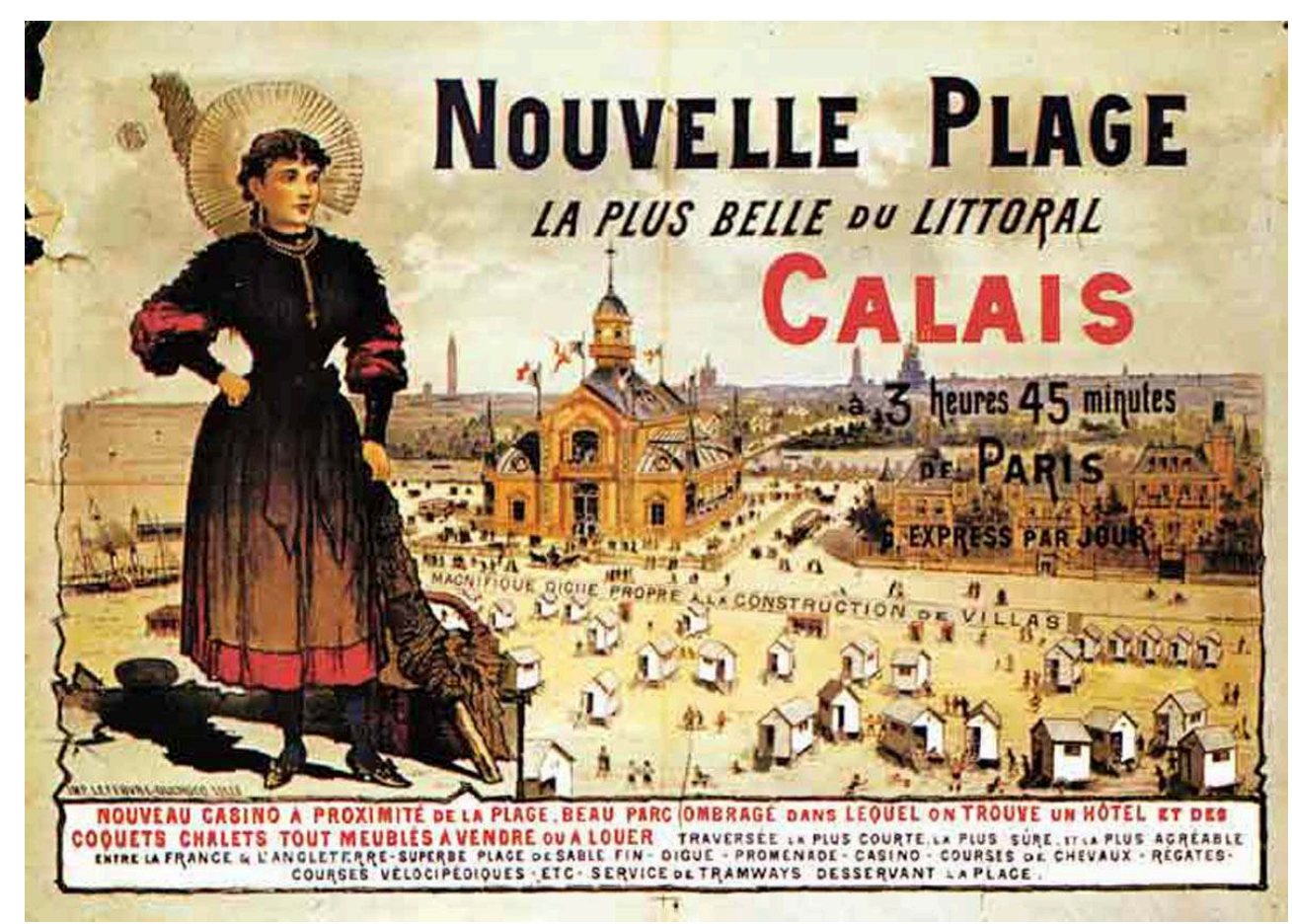

Calais (Pas-de-Calais). Affiche, s.d. [fin XIXe siècle ?]. Coll. part. Extrait de VILLES d'EAUX, 2002. Paris Imprimerie nationale Editions/Dexia, p. 30. 
2 Les réseaux de la villégiature s'organisent selon la nature des activités proposées, leur spécialisation médicale ou climatique mais aussi en fonction de leur accessibilité depuis les grands centres urbains et notamment Paris ${ }^{4}$ (fig. $\mathbf{n}^{\circ} \mathbf{1}$ ).

3 On pourrait donner à cette villégiature la même définition proposée par Alphonse Daudet dans son Tartarin sur les Alpes (1885) pour le tourisme. "Le tourisme est un ensemble de relations et de faits constitués par le déplacement et le séjour de personnes hors de leur lieu de domicile, en tant que ce séjour et ce déplacement ne sont pas motivés par une action lucrative quelconque ».

4 Le réseau urbain de la villégiature s'étend sur tout le territoire dans un espace relié par des mailles constituées par des voies de communication transportant des personnes. Chaque ville de villégiature ne représente pas seulement une unité autonome, un centre avec sa périphérie, mais une zone de condensation spécifique autour de points de croisement, les "échangeurs» des voies de communication. Séjourner en villégiature ne désigne pas le lieu statique de la "résidence", mais un espace dynamique d'échange, de circulation et de communication. Le nomade en villégiature, l'« étranger " à la station, est un "abonné » à ces points nodaux de l'espace-réseau, «branché » sur un réseau de services de santé, de confort et de loisirs. Mais la production de cet espace de villégiature suit-elle les mêmes règles que celles de l'espace de production de la ville industrielle?

5 Les articles de ce numéro examineront successivement les conditions de la mise en place et de l'évolution de ces réseaux de loisirs. Les réseaux sociaux et culturels s'établissent à partir d'une propagande médiatique qui se déroule selon des processus similaires sur tout le territoire. Le réseau des transports maritimes, routiers, ferroviaires et aériens réorganise l'espace $:$ il « ordonne » les nouveaux espaces urbains selon les flux migratoires et l'accessibilité de ces stations aux nouvelles infrastructures. Les sociétés immobilières ou hôtelières régissent les conditions d'occupation temporaire ou résidentielle sur ses nouveaux territoires. Des sociétés commerciales se spécialisent dans les services thérapeutiques et ludiques.

6 Les espaces de la villégiature se créent également à partir des réseaux personnels des «nomades ", suivant leurs relations sociales, culturelles ou territoriales et à partir des savoir-faire des animateurs et des promoteurs. Ces villes nouvelles de migrants établies aux marges du territoire, ouvertes aux nouvelles images et représentations mentales ${ }^{5}$, servent de creusets aux influences culturelles véhiculées par les maîtres d'œuvre et les maîtres d'ouvrage de toutes origines.

\section{La création des réseaux de loisirs}

7 Les industries de loisirs liées au tourisme sont contemporaines de la révolution industrielle et apparaissent plus tardivement en France qu'en Grande-Bretagne. D'après Marc Boyer, l'historien du tourisme, la « Révolution de Bath » permet dès le XVIIIe siècle à l'aristocratie de naissance de se mêler aux couches nouvelles enrichies par le commerce et l'industrie, sous prétexte de « prendre les eaux ». Ce modèle anglais de la saison mondaine dans les bathing places thermales s'étend à partir du dernier tiers du XVIIII siècle aux bords de mer des côtes anglaises puis au continent.

8 A partir du début du XIXe siècle, en quelques dizaines d'années, le thermalisme devient peu à peu un projet commercial : l'exploitation systématique des eaux minérales se 
développe sous le Premier Empire. Des villes s'édifient sur les modèles anglais de Bath autour de thermes antiques et de grands hôtels, ou de Brighton avec des établissements de bains et des casinos déployant une architecture fonctionnelle et luxuriante. A cette époque, en France, Luchon, Plombières ou Aix-les-Bains voient le jour.

Sous la Restauration et la Monarchie de Juillet, le thermalisme entre dans une phase de renouveau: la duchesse de Berry lance la mode des bains de mer à Dieppe, première station balnéaire française ${ }^{6}$. Par la suite, la présence de Napoléon III et de l'impératrice Eugénie dans les villes d'eaux ont contribué largement à l'essor du thermalisme et au développement des cités balnéaires. Les retombées de ces cures sont bénéfiques non seulement pour les villes élues mais pour l'ensemble des stations. A partir de 1856, Napoléon III passe onze saisons dans trois villes d'eaux différentes : Plombières ${ }^{7}$, SaintSauveur ${ }^{8}$ et Vichy ${ }^{9}$.

Le thermalisme est pour Napoléon III un champ d'essai exemplaire pour ses projets urbains. La mise en place de cette politique est contrôlée par la commission des eaux minérales qu'il met en place en 1854, composée notamment d'un médecin-inspecteur, d'un inspecteur en chef des mines et d'un architecte inspecteur des établissements sanitaires et thermaux de l'Etat, chargés de contrôler ce réseau des stations thermales et des premières stations balnéaires.

11 L'exploitation des sources ${ }^{10}$ et ensuite la reconnaissance des communes érigées en stations hydrominérales ou climatiques font l'objet d'une abondante législation pour bénéficier de cette nouvelle richesse économique et tirer de substantielles recettes: taxes de séjour, taxes sur les jeux ${ }^{11}, \ldots$

\section{L'invention thérapeutique et le réseau médical}

12 Le "prétexte » à la création de ces stations thermales, balnéaires ou encore villes d'hiver est médical: il s'agit d'éloigner des miasmes de la ville. Au XIXe siècle, en France, le « changement d'air » est particulièrement recommandé aux citadins. Chaque station thermale est ainsi un enjeu de pouvoir entre les propriétaires des eaux, le médecin-inspecteur qui en réglemente l'administration et les autres médecins qui veulent garder la liberté de prescrire. La notoriété du médecin crée la station et développe sa renommée. Le médecin crée le besoin, découvre les sources, "fabrique " le produit approprié au traitement des maladies et impose ensuite à ses patients les stations les plus appropriées à leurs pathologies. De nombreux traités médicaux sont des monographies écrites par des médecins locaux. La scientificité affichée par leurs auteurs cache mal l'intérêt de ces derniers - à la fois agents et relais de la propagande et même parfois aussi directement actionnaires. 


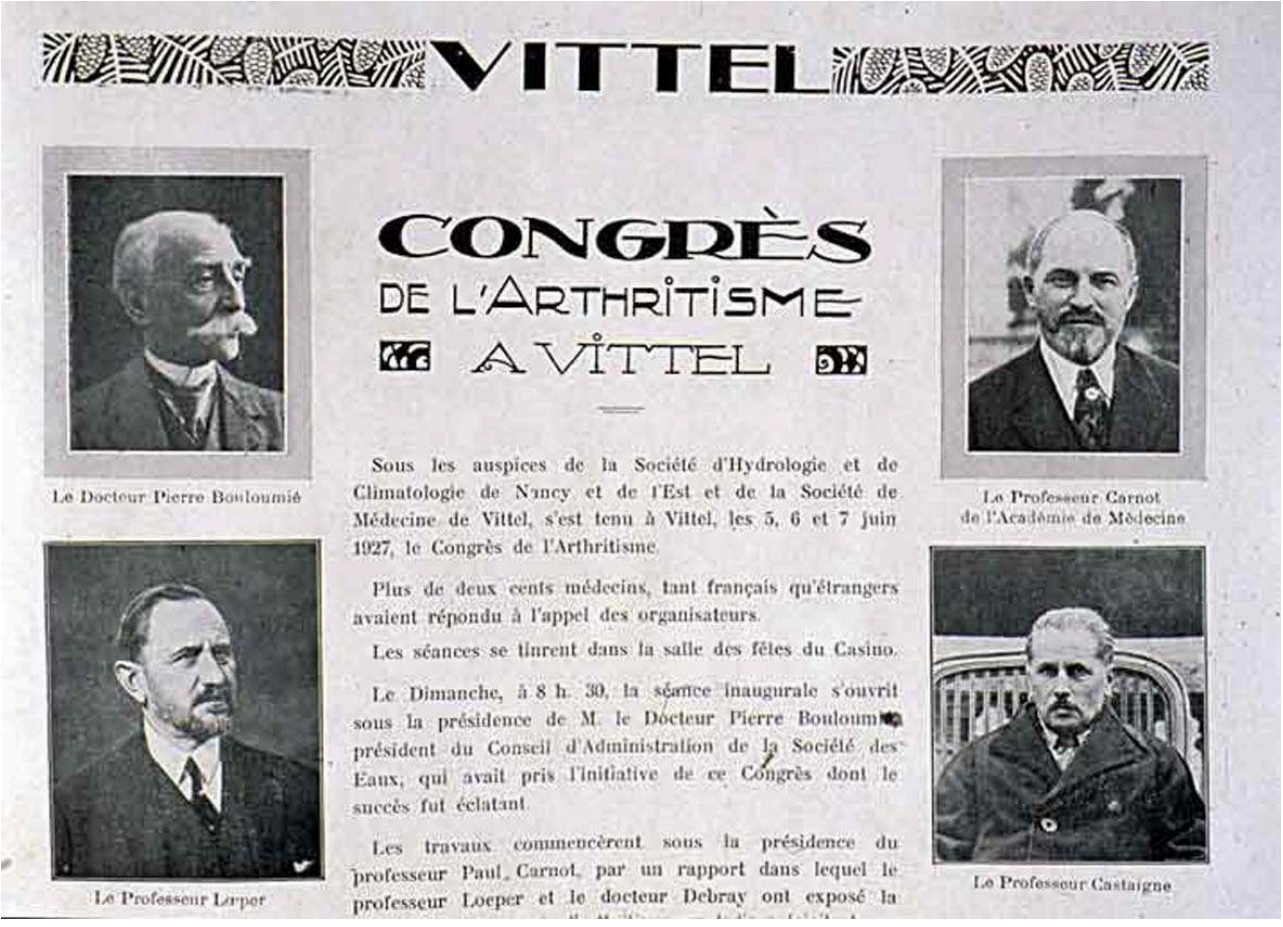

Congrès de l'arthritisme à Vittel. Extrait de vitTeL-JournaL, $7^{\text {er }}$ mai 1928. n.p ; Compte-rendu du congrès de 1927. Coll. part.

Repro. B. Toulier @ Droits réservés

A partir du milieu du XIXe siècle, la famille de médecins et notables Bouloumié « invente » et dirige la station thermale de Vittel : elle en orchestre la renommée pour le traitement de l'arthrite durant près d'un siècle. En 1927, à Vittel, le docteur Pierre Bouloumié, président du conseil d'administration de la Société des eaux est à l'initiative d'un congrès international sur l'arthritisme. Ce rassemblement de plus de deux cents médecins est placé sous les auspices de la Société d'hydrologie et de climatologie de Nancy et de la Société de médecine de Vittel. L'événement est relayé par le «VittelJournal », envoyé à de nombreux membres du corps médical et diffusé aux curistes ${ }^{12}$ (fig. $\mathbf{n}^{\circ} \mathbf{2}$ ). Ces congrès internationaux et les voyages d'études médicales dans les stations de cures relayent les enseignements et avis de l'Académie de médecine ${ }^{13}$ et les recherches de la Société d'hydrologie et de climatologie ${ }^{14}$. Des réseaux de médecins spécialisés en hydroclimatologie ou en thalassothérapie ${ }^{15}$ sont abonnés à la presse thermale et climatique ${ }^{16}$. 


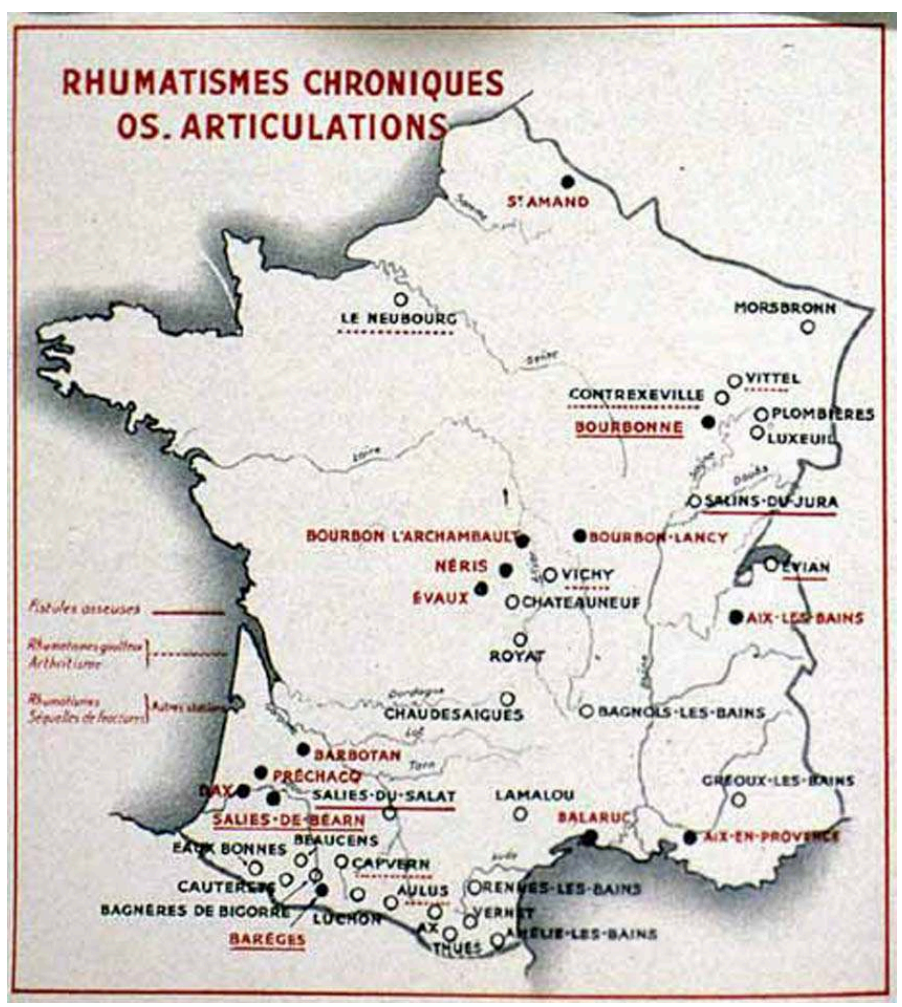

Carte des stations thermales traitant des rhumatismes chroniques des os et des articulations. Extrait LA théraPeutique thermale française. Paris : Centre national d'expansion du thermalisme, du tourisme et du climatisme, 1937, p. 52. Coll. part.

Repro. B. Toulier (c) Droits réservés

Des annuaires médicaux classent les stations selon les affections traitées et donnent des renseignements sur les sources, les établissements thermaux, les types de soins, les indications thérapeutiques et chirurgicales et leurs contre-indications, accompagnés de la liste des médecins consultants et spécialistes ${ }^{17}\left(\right.$ fig. $\left.\mathbf{n}^{\circ} \mathbf{3}\right)$. Le produit médical offert aux curistes est très segmenté et traite toutes les maladies.

Durant la saison, les stations les plus réputées accueillent les médecins parisiens qui suivent leur clientèle ou qui, comme elle, passent la saison d'hiver sur la Côte d'Azur ou dans les stations climatiques de montagne. Le réseau médical fidélise ses clients par la vente d'eaux minérales en provenance des stations dont la majorité est actuellement regroupée entre les mains de quelques grands groupes industriels ${ }^{18}$.

\section{La fièvre thermale et la ruée vers l'eau des affairistes}

Le Second Empire voit se développer les villes d'eaux de l'empereur et la fièvre thermale dépasse le cercle médical. Elle atteint celui des banquiers, des industriels et des particuliers qui investissent dans la création et le développement des stations thermales, dont les eaux sont des «sources de richesses intarissables ». L'eau minérale est une "mine liquide qui s'épanche spontanément à la surface du sol». Durant la Troisième République, les frères Brosson lancent Châtel-Guyon, sous l'impulsion du banquier Brocard. En 1886, Maupassant, dans son roman Mont-Oriol ${ }^{19}$ relate les âpres luttes qui se livrent autour des sources de Châtel-Guyon, avec la «guerre des 
sondages» pour le captage de nouvelles sources. L'écrivain décrit les étapes du lancement de la station d' "Enval » avec l'achat des terres par une société au capital fictif géré par le banquier Andermatt qui transforme le village en station thermale : «Tenez, regardez ce village, ce pauvre village! J'en ferai une ville, moi, une ville blanche, des voitures, une foule de riches servie par une foule de pauvres; et tout cela parce qu'il m'aura plu, un soir, de me battre avec Royat, qui est à droite, avec ChâtelGuyon, qui est à gauche, avec le Mont-Dore, La Bourboule, Châteauneuf, Saint-Nectaire, qui sont derrière nous, avec Vichy qui est en face». Comme la ville industrielle, la compétitivité économique de la ville de villégiature dépend de la qualité de ses services, face à la concurrence d'autres villes attractives.

17 Jérôme Pénez analyse ici la mise en place de ces réseaux d'investissements dans les stations thermales au XIXe siècle et Sophie Cueille développe les stratégies de ces investisseurs à travers les études de cas des bords de ville de la région parisienne.

Pour les sites établis en bord de mer, le passage d'un lieu inconstructible et parfois insalubre à un lieu au goût du jour entraîne des enjeux spéculatifs exceptionnels, une ruée vers l'eau. La ville nouvelle d'Arcachon conçue pour Emile Péreire en $1864^{20}$ est un exemple parfait de cette fièvre spéculative décrite quinze ans plus tard par le géographe Elisée Reclus. "Arcachon ressemble d'une manière étonnante à ces villes américaines qui s'installent en pleine forêt vierge et projettent leurs rues dans la solitude, sans se préoccuper des obstacles. En se promenant sur le bord de la petite mer intérieure des Landes, ceux qui connaissent la Louisiane pourraient se croire transportés à Madisonville, à la Passe-Christiane, à Pascagoula : ce sont les mêmes constructions éparses entourées d'arbustes, les mêmes collines couvertes de pins, le même bassin aux longues plages basses. De tous les côtés, on voit s'élever de nouvelles constructions, des chalets suisses, des manoirs gothiques, des pavillons mauresques, et jusqu'à des pagodes hindoues et des temples chinois. La ville grandissante transforme graduellement la forêt en un parc de plaisance au moyen d'allées sinueuses qu'elle projette au loin dans toutes les directions. La construction des maisons, la mise en culture des jardins, le percement des routes, et tous les embellissements exigent un si grand nombre d'ouvriers que de proche en proche le taux des salaires augmente dans les localités environnantes, et jusqu'à Bordeaux. En même temps, la valeur des terrains s'accroît dans une proportion rapide, et des propriétaires qui retiraient un bien maigre profit de leurs forêts vendent maintenant le mètre carré de sable aussi cher que s'il était situé sur la grande rue d'une cité populeuse ${ }^{21} \ll$.

\section{Du réseau des médias au réseau des promoteurs}

Le succès de la station repose sur une série d'acteurs successifs : les découvreurs, puis les fondateurs-promoteurs. La propagande entretenue par l'accueil de célébrités assure le degré de notoriété et de renommée de la station nécessaire pour attirer une clientèle avide de prestations médicales sophistiquées et de produits touristiques séduisants. 
Figure 4

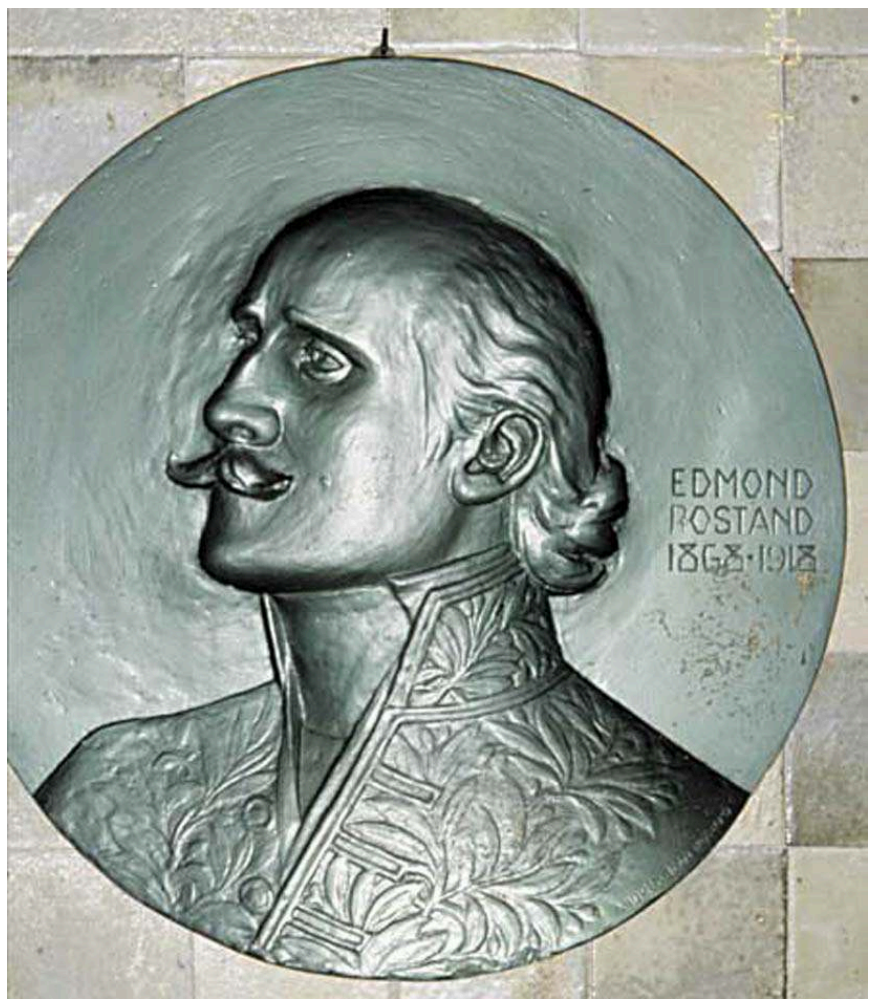

Bas-relief d'Edmond Rostand (1868-1918) par André-Jean Rostand. Cambo-les-Bains (PyrénéesAtlantiques), villa Arnaga.

Phot. B. Toulier (c) Droits réservés

Les découvreurs sont souvent des artistes ou des écrivains qui révèlent la station à leurs contemporains et au milieu parisien comme Maxime Leroy (1873-1957) ou Paul Margueritte (1860-1918) à Hossegor (Landes) ${ }^{22}$, George Sand (1804-1876) à Tamaris près de Toulon (Var) ou Edmond Rostand (1868-1918) à Cambo-les-Bains (PyrénéesAtlantiques) (fig. $\left.\mathbf{n}^{\circ} \mathbf{4}\right)$. Très vite, les artistes disparaissent, chassés par une deuxième vague d'occupants propagandistes : journalistes, directeurs de journaux, chroniqueurs et rédacteurs de guides parfois payés par le promoteur de la station jouent un rôle clé dans la promotion touristique. 
Figure 5

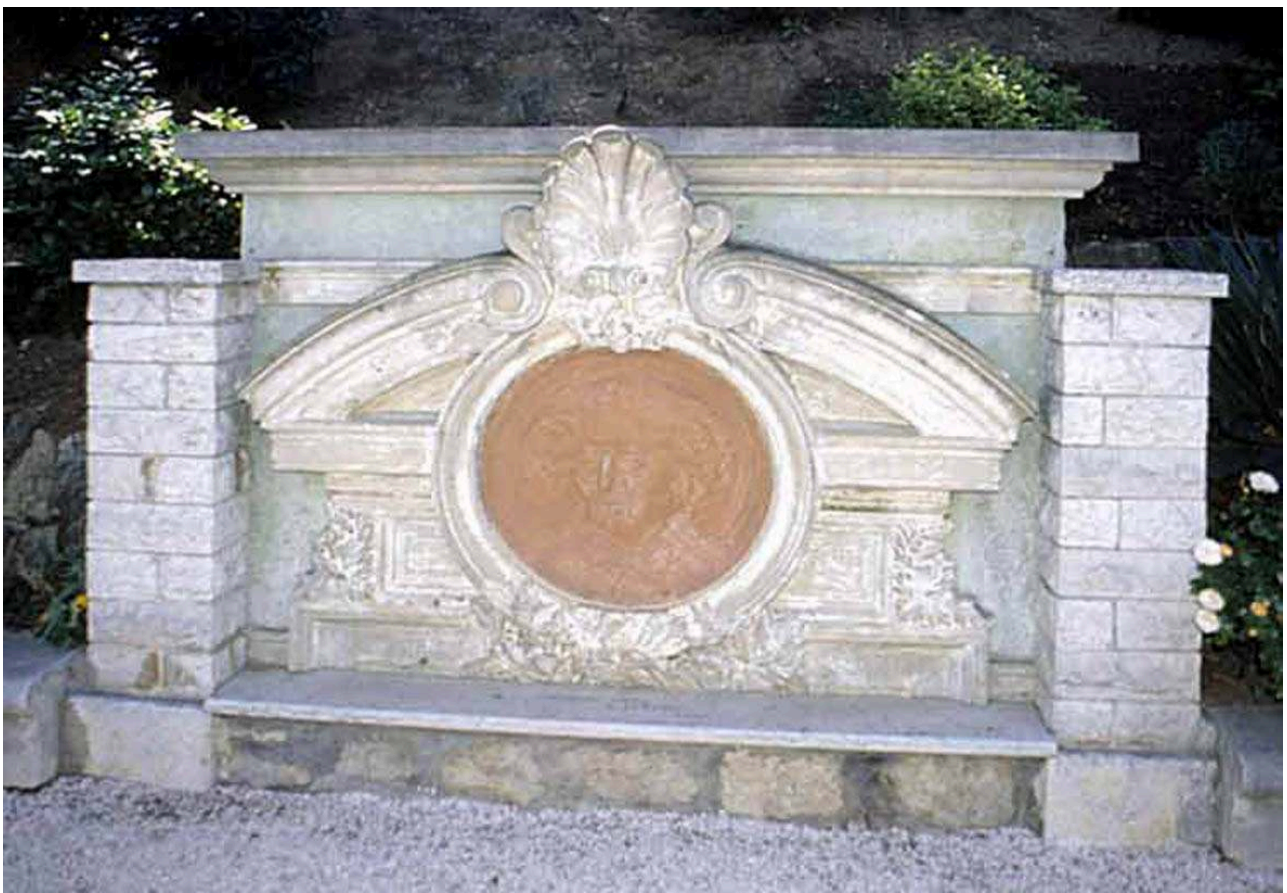

Bas-relief en terre cuite de George Sand (1804-1876). Placé en 1891 sur la bastide où elle séjourna. Aujourd'hui au musée de La Seyne-sur-Mer.

Phot. B. Toulier (c) Droits réservés.

21 A Tamaris, Paul Joanne, rédacteur de guides touristiques relaye rapidement la réputation de la station « découverte " par l'écrivain George Sand (fig. $\mathbf{n}^{\circ}$ 5). En 1861, celle-ci avait effectué un séjour hivernal dans l' " oasis » de Tamaris pour bénéficier des principes thérapeutiques du climat et des effluves balsamiques de sa forêt de pins. La rusticité de cette "île» sauvage et exotique lui inspirait la rédaction d'un roman éponyme, Tamaris, qu'elle publiait l'année suivante. Elle assure ainsi le lancement de la future station qu'elle déclare "plus belle que le Bosphore». Paul Joanne reprend aussitôt ce renom prestigieux créé par l'écrivain dans son guide De Lyon à la Méditerranée, publié en $1866^{23}$. 


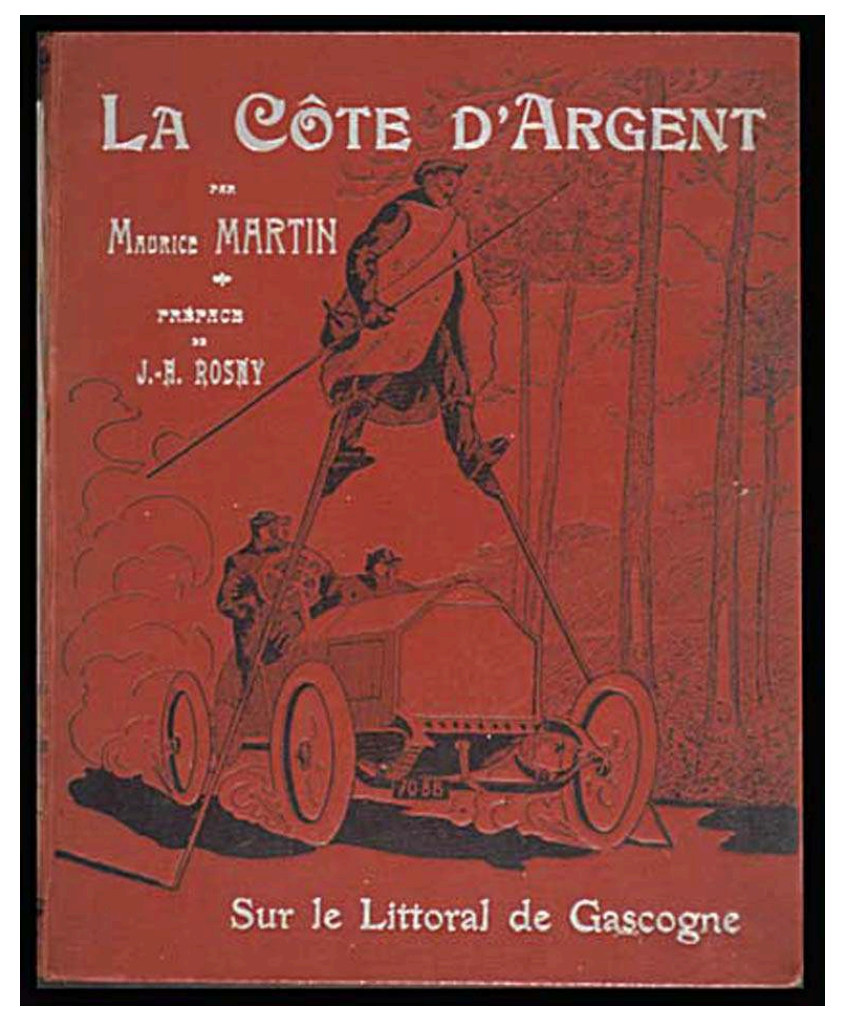

Couverture de l'ouvrage de MARTIN Maurice. D'Arcachon à Biarritz à travers les landes. La Côte d'Argent, partie méridionale.Bordeaux : Imprimerie G. Gounouilhou, 3e édit., 1907. Coll. part.

Repro. B. Toulier (c) Droits réservés.

En référence à la Côte d'Azur, la côte landaise, entre l'embouchure de la Gironde et celle de l'Adour, est rebaptisée en 1905 par le journaliste Maurice Martin La Côte d'Argent, dont «la vague éternelle, tantôt calme tantôt courroucée, vient déposer sa frange argentée au pied des dunes immaculées "! ...La «Côte d'Argent parce que, dans cet éblouissant décor, ... l'Atlantique fait sans cesse jaillir comme une blanche et colossale étincelle de son contact avec le vieux monde $!^{24} »\left(\right.$ fig. $\mathbf{n}^{\circ}$ 6). Ces chantres de la villégiature enjolivent la réalité et inventent une histoire fabuleuse des chaînes de stations, à la manière des épopées légendaires de la conquête de l'Ouest américain. Les directeurs anonymes d'agences de voyages sont aussi d'éminents relais, propagateurs de renommée, souvent perdus entre les services commerciaux des grandes compagnies de transports, les rédacteurs de guides et les directeurs des stations. Ceux-ci les « invitent » régulièrement à visiter leurs établissements. Ainsi, les 24 et 25 juin 1927 la Société des eaux de Vittel accueille les directeurs des agences européennes les plus prestigieuses au Grand Hôtel ${ }^{25}$ où ils visitent l'établissement thermal, en clôture à leur excursion dans les stations thermales de l'Est de la France, « organisée » notamment par la Compagnie ferroviaire des chemins de fer de l'Est ${ }^{26}$.

Une fois lancée, la station est prête à accueillir son fondateur-promoteur, au destin providentiel. L'entrepreneur-promoteur, investisseur-spéculateur est le père fondateur de la ville d'eaux comme l'architecte-paysagiste Henry Martinet à Hendaye-Plage, Roger Eluère à Hossegor ${ }^{27}$, René Brouard à Sables-d'Or-les-Pins, Louis Lajarrige à Escoublac-La Baule, Armand Peugeot à Morgat, Ali Pacha à Tamaris ou Ernest Dufayel à Sainte-Adresse ${ }^{28}$. 


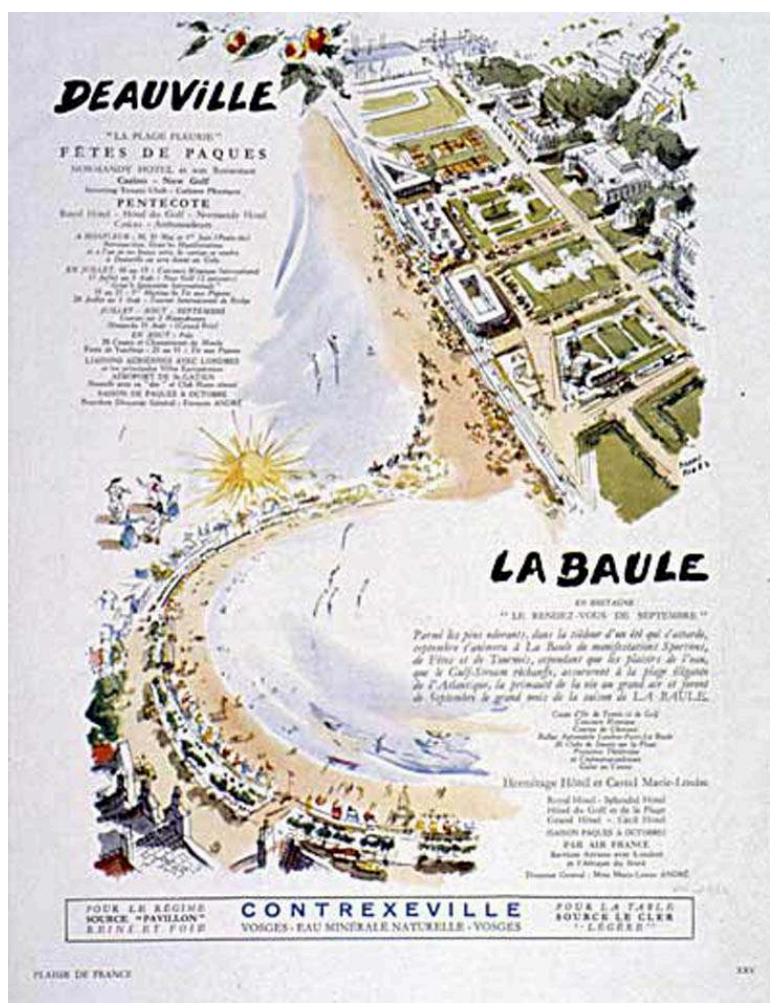

Page de publicité pour les stations de Deauville et de la Baule. Extrait de Plaisir de France, avril 1952. La majorité des équipements des deux stations font partie de l'empire de François André. Coll. part. Repro. B. Toulier @ Droits réservés

Ces « industries» thermales ou climatiques sont pour une large part gérées par des particuliers qui exploitent directement ou par l'intermédiaire de sociétés : sociétés immobilières, société d'exploitation des eaux, ... Au début du XXe siècle, les collectivités et l'Etat, qui possède six établissements thermaux civils, exploitent par un système de concessions à des sociétés fermières à but commercial. Ce système économique des loisirs regroupe trois branches d'activité qui peuvent parfois s'associer en des réseaux financiers complexes et créer des empires comme celui fondé par François André (fig. $\left.n^{\circ} 7\right)^{29}$. 


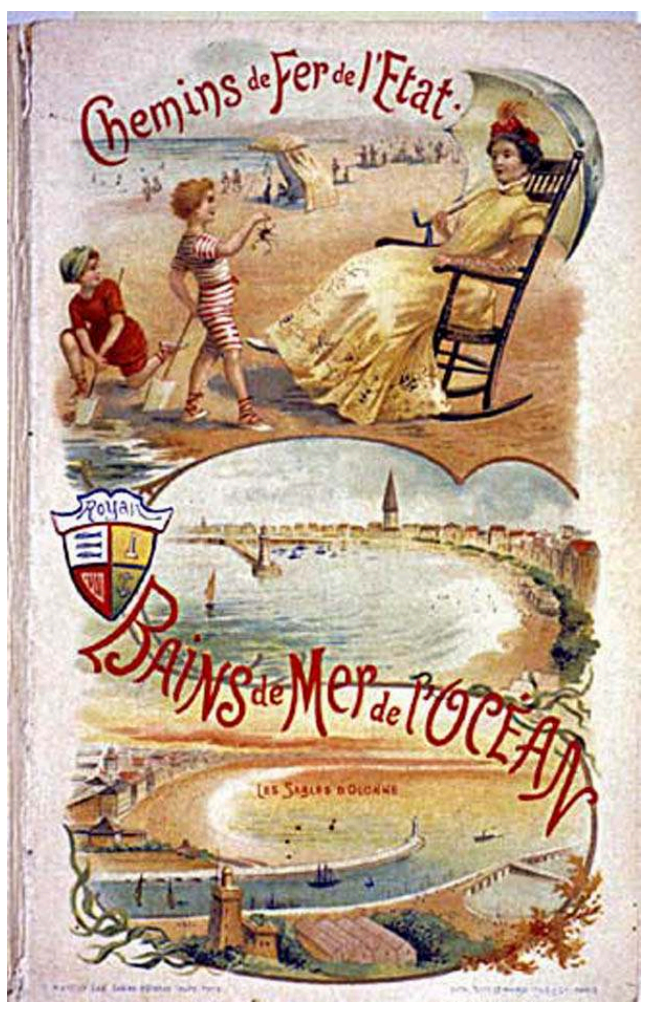

Couverture du livret-guide des BAINS DE MER DE L'OCÉAN. LES SABLES-D'OLONNE, édité par la Compagnie des chemins de fer de l'Etat, 1900. Coll. part.

Repro. B. Toulier (c) Droits réservés

La branche des voyages s'étend aux compagnies de transports. La branche immobilière organise et gère les séjours temporaires dans les hôtels, les palaces, les villas et les lotissements privés (fig. $\mathbf{n}^{\circ} \mathbf{8}$ ). Enfin, diverses sociétés spécialisées exploitent les produits commerciaux de consommation à but thérapeutique ou de loisirs.

\section{Le nouveau réseau spatial}

Ce nouveau réseau économique de ces stations de villégiature s'inscrit dans l'espace et dans le temps et structure le territoire. Ces stations se caractérisent par le caractère saisonnier de leur activité économique. L'implantation et le développement de ces agglomérations nouvelles dépendent de la nature des activités et leur degré d'accessibilité. Elles répondent à une logique de gestion du flux des déplacements, selon les diversités et les complémentarités de la chaîne des transports, du lieu d'origine à la destination, en comptant sur les nœuds de transbordement.

Le point de convergence entre ces différentes stations et les réseaux qui les relient est la création d'un maillage du territoire pour l'occupation d'espaces vierges consacrés à des activités originales, non directement productives, à l'opposé de la ville industrielle : lieux d'un autre mode de vie, hors des contraintes de la ville conventionnelle et traditionnelle. Les stations se développent selon des logiques de création en bassins ou en chaînes, continues ou discontinues selon leur pouvoir d'attraction et le jeu de la concurrence. Elles s'agglomèrent autour de pôles structurants dont les tentacules s'étendent parfois en chapelets autour de stations-satellites comme Biarritz avec Saint- 
Jean-de-Luz, Hendaye, Anglet, Guéthary et Bidart ou La Baule avec Pornichet et Le Pouliguen $^{30}$.

\section{Les réseaux des stations} de certaines stations comme Arcachon ou Royan, reliés à Bordeaux, Trouville au Havre, Dinard à Saint-Malo ou Tamaris à Toulon. Le développement des stations balnéaires est lié aux progrès de la navigation à vapeur et surtout au chemin de fer puis à l'automobile et, dans une moindre mesure à l'aviation. Mais de nombreux centres balnéaires existent avant l'arrivée du train : il n'y a pas d' "axiome d'indispensabilité " entre desserte ferroviaire ou apparition de l'automobile et développement balnéaire, comme le démontre Marc Boyer ${ }^{32}$.

Vers 1847-1848, l'ouverture des lignes de chemin de fer Paris-Le Havre et Paris-Dieppe déclenche les premiers engouements pour les bains de mer auprès de la bourgeoisie parisienne. A partir du Second Empire et surtout de la Troisième République, les stations s'accrochent au chemin de fer " comme les feuilles d'un arbre à ses branches ". Pour la saison des bains de mer de l'année 1850, les premiers « trains de plaisir » relient la capitale aux stations du Havre et à Dunkerque; le procédé s'étend les années suivantes aux stations de Calais, Dieppe et de Boulogne. Le premier train de plaisir arrive en gare de Saint-Malo le $1^{\mathrm{er}}$ août 1866, deux ans seulement après l'inauguration de la gare. Vers 1870 , des voyages circulaires sont organisés par les compagnies comme les excursions sur les côtes de Normandie et de Bretagne ou les bains de mer de la Manche $^{33}$. D'autres initiatives commerciales facilitent le voyage comme les billets 
valables plusieurs jours émis à partir des années 1865 en direction des stations balnéaires ${ }^{34}$.

Figure 9

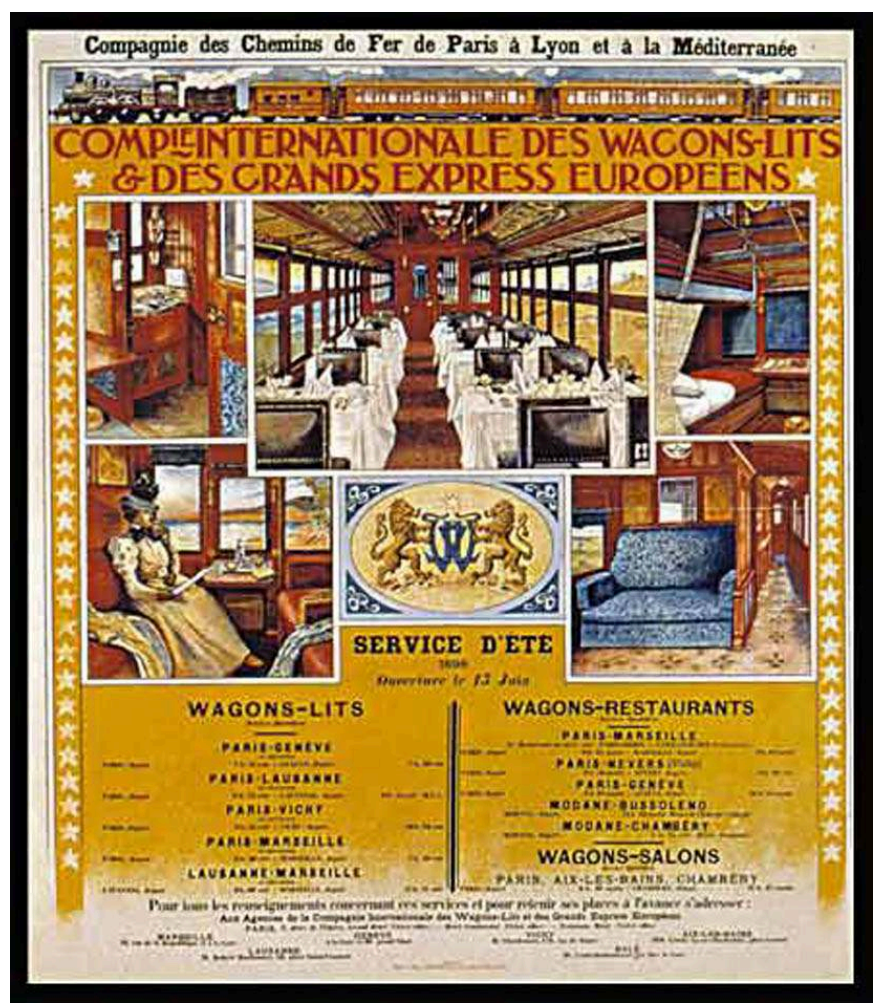

Affiche de la Compagnie des chemins de fer de Paris à Lyon et à la Méditerranée, pour la Compagnie internationale des wagons-lits et des grands express européens. Saison d'été 1898. Coll. part.

Repro. B. Toulier @ Droits réservés

34 A partir de la seconde moitié du XIXe siècle jusqu'en 1914, le flux des voyageurs à destination des stations thermales et balnéaires est rémunérateur. La clientèle de privilégiés effectue des trajets sur de longues distances en première classe, voire en seconde mais jamais en troisième. Des trains spéciaux sont mis en place durant la saison estivale, de juin à octobre, trains directs et de luxe. Plusieurs compagnies comme la Compagnie internationale des wagons-lits jouissent d'une grande réputation (fig. $\mathbf{n}^{\circ}$ 9). A partir de Paris, des trains spéciaux sont affrétés à destination des stations. Le Sud-Express, composé de wagons-lits, de salons et de restaurants rejoint les stations balnéaires de la Côte d'Argent et les stations thermales des Pyrénées selon une fréquence tri-hebdomadaire. A partir de 1910, il est en correspondance avec le PyrénéesCôte d'Argent-Express. On trouve un Londres-Paris-Vichy ou le Thermal-Express à destination de Vichy, Châtel-Guyon, Royat, La Bourboule et Le Mont-Dore.

Les politiques touristiques ferroviaires furent multiformes et celle suivie par la Compagnie du Midi, décrite par Christophe Bonneau, nous offre certainement le modèle le plus cohérent et le plus achevé, grâce au volontarisme des conceptions de ses dirigeants $^{35}$. Des Pereire, fondateurs durant le Second Empire, au directeur Jean-Raoul Paul, qui incarne une nouvelle ère managériale au début du siècle, la politique touristique de la compagnie se plaça sous le signe de la continuité : importance des opérations immobilières au titre du domaine privé, multiplication des initiatives 
tarifaires, organisation de trains de plaisir complétée dans l'entre-deux-guerres par des services d'autocars ${ }^{36}$.

L'arrivée de la traction électrique améliore les dessertes urbaines et favorise l'implantation de nouveaux quartiers de villégiature. La ligne du P.C.L. (PierrefitteCauterets-Luz), exemple de l'introduction et du développement de la traction électrique pour prolonger la voie Midi-Lourdes-Pierrefitte est ouverte en 1898 pour assurer la desserte de la ville d'eaux de Cauterets. Elle joue un rôle pionnier dans ce type d'infrastructure thermale de transport ${ }^{37}$.

Aux Sables-d'Olonne une ligne de tramway dessert au début du XXe siècle l'ancien casino, passe le long du remblai pour accéder ensuite au nouveau casino. Les funiculaires abolissent la barrière des dénivellations. Le funiculaire de La Raillère à Cauterets relie les bains de la vallée à ceux de la montagne. Les hauts de falaises s'urbanisent comme au Tréport-Terrasse et de nouvelles activités climatiques et sportives sont pratiquées sur les hauteurs, accessibles depuis les fonds de vallée comme au Mont-Revard à Aix-les-Bains ${ }^{38}$.

Les liaisons routières se développent également sous le Second Empire avec la création de routes thermales. Dans les Pyrénées, Napoléon III ordonne la création d'un réseau de routes pour relier les stations de Bagnères-de-Bigorre, Luchon, Barèges, Cauterets, les Eaux-Bonnes et les plus petites stations de Labassere, Gazost, Saint-Sauveur... séparées par des montagnes et des cols élevés.

A partir du début du XXe siècle, l'usage de l'automobile révolutionne l'accès aux stations. Dès 1907 par exemple, le journaliste Maurice Martin propose, avec l'aide du Touring Club de France et de l'Automobile Club de France la création d'une route le long du littoral, entre Arcachon et Biarritz, à travers les landes. "...On y fera de la vitesse tant qu'on voudra; de l'étape aussi, de la charmante, vivifiante étape, voire du camping... Quelques belles stations de luxe, sur un littoral magnifique, rendront, à ce point de vue, de grands services, et ces stations seront la conséquence inévitable d'une route automobile » (J.-H. Rosny, préface de Maurice Martin, La Côte d'Argent, 1907).

- Les compagnies de chemin de fer investissent également dans l'automobile en créant des services relais d'autocars. En 1911, la compagnie Paris-Lyon-Marseille (P.L.M.) organise un service de transport automobile d'Evian-les-Bains à Nice. Deux ans plus tard, des correspondances automobiles relient Vichy et Châtel-Guyon par ClermontFerrand et Royat avec le Mont-Dore et La Bourboule. Ce sera la "route thermale d'Auvergne». Initié par la compagnie P.L.M., le principe des routes touristiques automobiles est bientôt repris par toutes les autres compagnies de chemin de fer.

Mais le relais rail-route fonctionne également sur le mode de la concurrence. Durant l'entre-deux-guerres, l'automobile supplante le chemin de fer pour l'accès à certaines stations $^{39}$. La gare de la nouvelle station de Stella-Plage créée pour l'automobile, ouverte en 1922 sur la ligne Berck-Le Touquet, cessera son activité dès 1926. Malgré la carte de la complémentarité jouée par les compagnies de chemin de fer, on assiste déjà aux premières victoires de la route sur le rail. Cependant le rail a encore de beaux jours pour acheminer le curiste ou le baigneur sur de longues distances, notamment en direction des 2000 kilomètres de côtes et les 600 plages desservis à cette époque par le réseau de l'Etat. 


\section{Le réseau comme moyen de production du territoire} aux établissements thermaux de l'Etat. L'architecte organise l'espace urbain qui intègre le quartier thermal à la cité préexistante à partir de la gare, départ d'un réseau viaire en patte d'oie qui ceinture la ville et descend jusqu'aux bords de l'Allier. Cette rive est aménagée en parc et rejoint le parc thermal des sources. Pour le quartier thermal, Isabelle conçoit à côté des trois établissements thermaux et industriels de nouveaux espaces ludiques et d'animation avec des jeux de paume, de quilles, de toupies, un tir et un casino avec théâtre et salle de bal. L'exécution du projet est un compromis entre cette conception théorique et la réalité économique résultant des contraintes spéculatives.

45 A la fin du XIXe siècle, la station thermale se définit comme une ville réduite en miniature, autour de son parc sur lequel donnent les principaux équipements publics et les chalets d'habitation, comme à Martigny-les Bains ${ }^{40}$.

La ville de Vernet-les-Bains est un des modèles-types de ces stations. «Une société financière est passée par là et, procédant à coups de millions, elle a formé le projet de créer là une ville d'eaux modèle, à la fois station d'été et station d'hiver. Le projet est actuellement à peu près réalisé. La ville thermale forme un domaine entièrement clos qui va se perdre jusque dans les montagnes qui ferment la vallée. Elle se compose d'un parc avec sa pièce d'eau et sa cascade, le tout gracieusement dessiné, d'un casino, d'un établissement thermal, d'une quinzaine de villas disséminées dans le parc et destinés à être louées en entier ou par parties aux baigneurs qui viennent s'installer avec leur famille, de cinq à six grands hôtels, d'un jardin merveilleux, d'une vacherie pour la cure du petit lait, d'un gymnase pour les enfants » (Gazette des Eaux, 15 septembre 1881). 
Figure 10

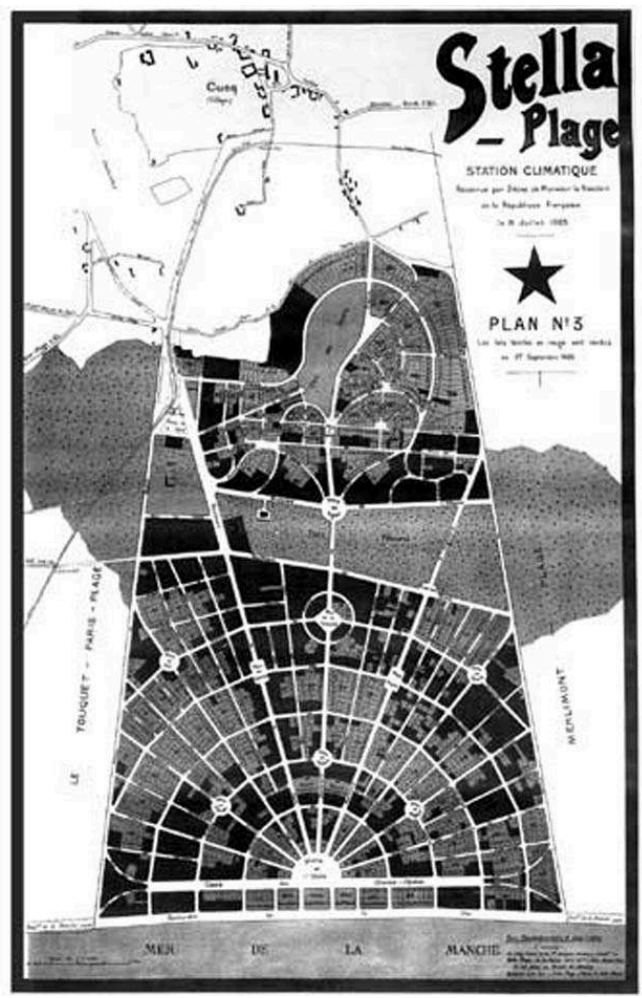

Stella-Plage (Pas-de-Calais). Projet de Charles Plumet, vers 1925. Archives nationales, Centre des archives d'architecture. Extrait de VILLES D'EAUX, 2002. Paris : Imprimerie nationale Editions/Dexia, p. 32.

47 Les villes nouvelles s'orientent habituellement à partir de l'axe structurant créé par la liaison fonctionnelle entre la gare et le casino ou l'établissement de bains. L'axe obligé du bord de mer se combine le plus souvent avec une hiérarchie de la voirie, à l'image d'une ville régulière, comme à Deauville. Durant le dernier quart du XIXe siècle, le Paris haussmannien est le modèle de référence pour de nombreuses stations balnéaires de l'Ouest. Les paysagistes anglais et le concept de la cité-jardin influenceront à leur tour les villes nouvelles, à partir de la fin du XIXe siècle et durant la première moitié du XXe siècle. Au Touquet Paris-Plage, les architectes Charles Garnier et Thomas Cutler, assistés de paysagistes anglais conçoivent en 1894 pour un public anglo-français une extension de la ville, Mayville pour l'homme d'affaires anglais John Robinson Whitley ${ }^{41}$. Le projet anglais, refusé par les habitants du Touquet, inspire le plan de la proche station d'Hardelot. En 1914, le plan de la nouvelle ville de Stella-Plage de Charles Plumet marie le plan radioconcentrique et régulier de la station de villégiature du Second Empire (Maisons-Lafitte, Cabourg ${ }^{42}$ ) avec le plan paysager au tracé sinueux de la ville d'hiver (Arcachon), située en retrait du front de mer à l'abri d'une pinède (fig. $\mathbf{n}^{\circ}$ 10). Mais la station fondée ex nihilo est rare. Plus fréquemment, la ville littorale s'organise autour de schémas d'urbanisme unipolaires ou multipolaires. Le centre-ville est relié à un ou plusieurs quartiers balnéaires organisés autour d'une ou plusieurs plages. 


\section{Le processus d'urbanisation et le lotissement balnéaire}

La possession du rivage touche au domaine maritime public géré par l'Etat. A partir de l'ordonnance royale de 1825 , les préfets concèdent une partie du rivage après une enquête commodo et incommodo. A Hendaye, l'Etat cède en 192059 hectares de terrains en bordure de plage et dans la baie de Chingoudy, à charge d'endiguement par la société immobilière. Une partie de ce domaine maritime relève aussi du domaine militaire, avec contraintes et interdictions de construire. Le quartier balnéaire doit parfois se développer hors les murs, constitué de bâtiments éphémères construits en bois, tradition qui se perpétue notamment dans le nord de la France, le long de la Manche. A Calais, en 1892, l'Etat cède à la ville l'usage d'une partie de la plage, à l'extérieur des fortifications, accessible par le tramway depuis la gare. L'année suivante, la commune passe une convention avec un certain E. Bresson pour la construction d'un casino en bois et brique. Le concessionnaire s'engage également à construire à ses frais la digue et une partie de la route qui dessert le casino et des villas qu'il doit construire sur le front de mer pour les baigneurs.

Le processus d'urbanisation et le passage du domaine public au domaine privé présentent un schéma commun aux villes thermales et balnéaires. Le lotissement, procédure de division du sol par lots en vue de la vente, de la location ou de la concession est une procédure fréquemment employée dans le passage du site vierge au site urbanisé des villes d'eaux. Plus d'un siècle avant la France, l'Angleterre expérimente le système du lotissement paysager pour l'aménagement des stations thermales (Bath, Royal Crescent de John Wood en 1769) et de ses quartiers urbains périphériques (Londres, Regent's Park de John Nash, achevé en 1838). Le modèle du lotissement paysagé, importé en France via les villes nouvelles de la couronne parisienne (Le Vésinet, Maisons-Laffitte, Enghien-les-Bains) séduit nombre d'investisseurs de stations balnéaires, notamment sur les côtes de la Manche et s'épanouit durant l'entre-deux-guerres sur la majeure partie du littoral. 


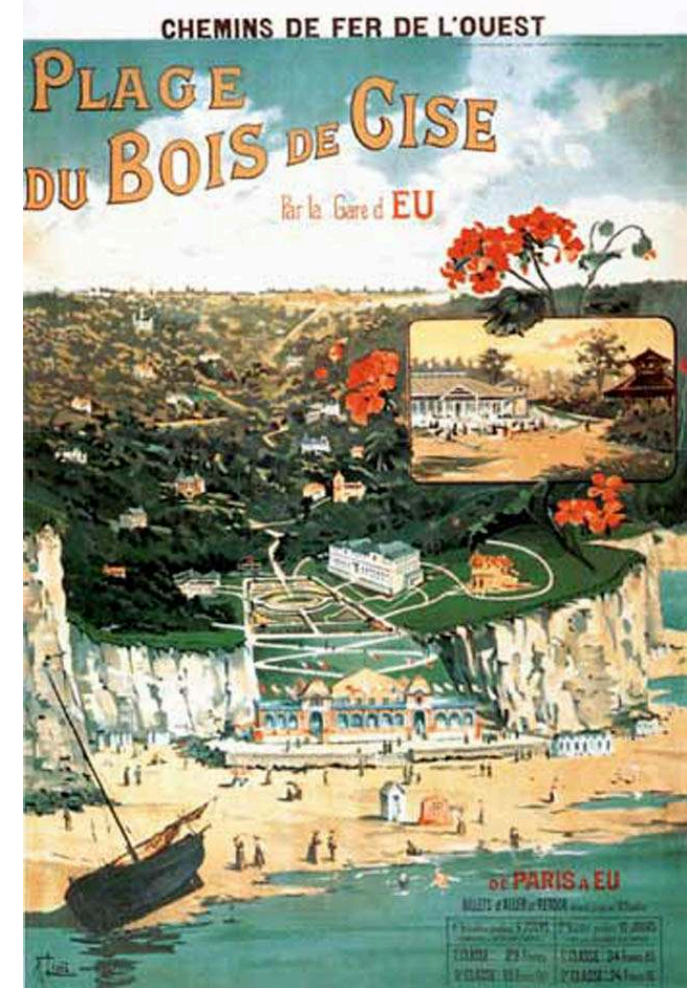

Le Bois-de-Cisse à Ault (Somme). Affiche de la Compagnie de chemin de fer de l'Ouest, vers 1899-1900. Extrait de VILLES D'EAUX, 2002. Paris : Imprimerie nationale Editions/Dexia, p. 31.

Les groupes d'habitations et les lotissements créés par des associations, des sociétés ou des particuliers sont soumis à l'obligation d'un plan d'aménagement approuvé par le préfet. Pour séduire les actionnaires du lotissement, architectes et promoteurs proposent l'établissement de bâtiments publics ambitieux. En 1898, le promoteur du Bois-de-Cisse Jean-Baptiste Theulot vante les mérites de sa nouvelle station par l'édition d'une affiche de propagande. Toutes les constructions-phares de cette petite cité y figurent, idéalisées : digue-promenade, hôtel, église et casino autour d'un grand parc (fig. $\left.\mathbf{n}^{\circ} \mathbf{1 1}\right)$.

\section{Les plans d'extension et d'embellissement des stations}

51 L'Etat ne tente de maîtriser ce phénomène d'urbanisme sur l'ensemble du territoire que bien tardivement, à partir du début du XXe siècle. La loi Cornudet du 14 mars 1919 (modifiée et complétée le 19 Juillet 1924) joue un rôle décisif pour les plans d'extension et d'aménagement des villes d'eaux. La loi prévoit l'obtention d'un label pour les «stations balnéaires, thermales et touristiques ». Les communes érigées en stations de climat, de bains ou de promenade doivent se doter d'une «chambre d'industrie thermale, climatique ou touristique » désignée par l'administration, qui seconde la municipalité.

52 Les plans d'extension et d'embellissement des stations se multiplient. A titre d'exemple on peut énumérer pour les stations thermales, sans souci d'exhaustivité : Lamalou-les- 
Bains (1920-1923 par l'architecte Tassin), Aix-les-Bains (1921-1927 par l'architecte Duquesne), Néris-les-Bains (1921-1923, par l'ingénieur Mercier), Salins (1922), Vittel (1926-1928 par l'architecte-urbaniste Jaussely), Châtel-Guyon (1930), Contrexéville (1930-1933 par les architectes-urbanistes Alex et Pierre Fournier), Royat (1931-1934), Luxeuil-les-Bains (1931-1936 par l'ingénieur-géomètre Mourgeon), Bourbon-Lancy (1934 par Marcel Génermont), Cauterets, Barbazan (1935 par l'architecte-urbaniste Dervaux), Vichy (1936-1939 par l'architecte-urbaniste Gaston Bardet)... Les architectes de la Société Française d'Urbanisme et les anciens élèves de l'Ecole des Beaux-Arts triomphent dans l'établissement de ces plans d'embellissement des villes d'eaux, seul secteur où l'application de la loi Cornudet enregistre un succès. Il reste cependant à mieux déterminer, à travers les monographies, l'impact de ces projets sur le renouveau urbain.

Les fonctions du territoire et du paysage de villégiature sont différenciées et évoluent selon leurs usages sociaux. «Le paysage est une réalité culturelle car il est non seulement le résultat du labeur humain, mais aussi l'objet d'observation, voire de consommation. La culture joue ici le rôle d'un filtre variable d'un individu à l'autre, d'un groupe social à l'autre. Ce phénomène de ricochet est capital dans les paysages touristiques qui sont avidement regardés mais aussi profondément aménagés pour être mieux regardés ${ }^{43} »$.

\section{Les réseaux sociaux et culturels} sociales, aux cultures des migrants, hivernants et estivants. A ses débuts, la station de villégiature est destinée à une clientèle aristocratique puis bourgeoise ${ }^{45}$. Durant le Second Empire, les stations thermales et balnéaires sont le plus souvent investies par des « clans » de rentiers, aristocrates ou bourgeois, aux mœurs convenues, véritables castes qui s'élisent par cooptation pour investir la station. Ce sont elles qui font et défont les modes par un processus d'imitation et de distinction entre les stations et à l'intérieur de celles-ci . Les «migrants» se regroupent par affinités, par quartiers ou même en "colonie » suivant leurs origines géographiques et sociales. Les différences d'origine sociale expliquent les spécialisations des stations balnéaires à la fin du XIXe 
siècle, entre les stations «mondaines " et les " petits trous pas chers » destinés à une clientèle familiale. A partir des années 1930, la spécialisation s'accélère, avec l'avènement des congés payés à la fin de la décennie pour prendre toute sa signification durant l'après-guerre jusqu'à la massification des flux touristiques des années 1960 .

Les différentes « colonies » des stations, organisées selon les familles et les milieux, les groupes d'intérêt sociaux et économiques forment des réseaux de sociabilité et une clientèle aux ramifications complexes, parfaitement analysés par Jacques Tati dans Les vacances de Monsieur Hulot ${ }^{46}$.

Les promoteurs et les architectes des villes d'eaux s'attachent à construire une image rassurante et séductrice pour attirer ou répondre à une clientèle particulière ${ }^{47}$.

Les règles de sociabilité entre "étrangers " sont une des bases de la réputation de la station. La présence de célébrités prestigieuses, rois, reines, empereurs, impératrices, grand-ducs, diplomates, banquiers, hommes de lettres, gens du spectacle, est nécessaire pour lancer la station et maintenir sa réputation à chaque saison. Les «listes d'étrangers » sont publiées dans les gazettes locales à partir des registres des hôtels. Elles collationnent soigneusement le nom, la qualité, l'origine et l'hôtel dans lequel est descendu le curiste ou le baigneur de marque, accompagné de sa famille et de ses domestiques. Les cartes de visite des hôtes sont épinglées dans le hall des hôtels. Ces annonces tissent la trame des réseaux de sociabilité dans la station, réglant le rituel de la distinction, de l'emploi du temps de la journée selon les opportunités de rencontre dans les établissements de soins, sur les lieux de promenade, de distractions et de sports. L'introduction des sports et leur évolution sont des pratiques sociales réglées selon ces principes de distinction ${ }^{48}$.

L'architecture est un des lieux de distinction privilégiés où circulent et s'exercent les modèles culturels en usage dans la villégiature. La villégiature est le point de rencontre entre deux cultures, celle de la clientèle exogène, urbaine, touristique et celle d'une culture indigène, régionale et longtemps rurale. Les stations ne sont pas construites ou rarement construites par des aristocrates ou des bourgeois locaux mais par une élite urbaine composés de petits «cercles » allogènes, considérés comme des étrangers par les gens du pays.

61 Souvent placés aux marges du territoire, ces lieux aux caractères sociaux très affirmés développent de fortes identités. Ces espaces interlopes sont un des lieux privilégiés où se développent les régionalismes architecturaux "identitaires $»^{49}$. Lieux d'innovation, les stations sont, suivant les époques des lieux de réception des modèles internationaux et des références d'outre-mer : anglomanie, orientalisme, style « international » ${ }^{50}$.

Paradoxalement, ces lieux de villégiature sont pour certains anciens émigrants revenus au pays un lieu de retour à une culture conservatrice et rassurante, aux antipodes des cultures d'avant-garde( ${ }^{52} 53$. En fait, de la fin du XVIIIe siècle à la crise de 1930 , la majorité des stations est peuplée par une clientèle composée essentiellement de rentiers ou de propriétaires; les commerçants et industriels dynamiques sont l'exception. Au XIXe siècle, les stations de villégiature où s'exerce le voyage par curiosité et désœuvrement sont pour les rentiers des lieux souvent tournés vers le passé, lieux de gaspillage de temps et d'argent où les jeux du casino et les dépenses ostentatoires pour les constructions temporaires et éphémères sont des critères de distinction pour l'élite ${ }^{54}$. 
63 Comme il existe une géographie des lieux de production industrielle ou des espaces urbains, celle des espaces et des territoires de la villégiature organisés en réseaux est sans doute un des traits les plus caractéristiques de l'histoire de la civilisation des XIXe et XXe siècles. Au XXIe siècle une partie de ces stations deviennent de "vraies villes " pour une vie tout au long de l'année et un tourisme des quatre saisons, amorçant comme en Languedoc-Roussillon une "seconde révolution touristique ${ }^{55}$. Le littoral français, destination des vacanciers de toute l'Europe est aujourd'hui une vaste métropole linéaire organisée en réseaux, denses ou en "peaux de léopard». L'occupation des bords de mer est destructurée, de plus en plus consacrée à la monoactivité touristique, grande consommatrice d'espace au détriment d'une gestion intégrée des ressources et des patrimoines ${ }^{56}$.

\section{NOTES}

1. Voir le site Internet: ..Puis Projets et recherche, Programme du CNRS, UMR 22 sur l'architecture de la villégiature.

2. TOULIER, Bernard. Architectures des villes d'eaux. Stations thermales et balnéaires. Villes d'eaux des Pyrénées occidentales. Patrimoine et devenir. Clermont-Ferrand, Institut d'études du Massif Central, 1996, p. 23-36

3. Voyages en France. Accès à 3000 imprimés et à 6000 images numérisés. Voir notamment l'accès géographique et le parcours thématique sur la naissance du tourisme: transports et tourisme au XIXe siècle, tourisme thérapeutique, montagne, bains de mer, tourisme sportif.

4. La « nouvelle plage » située au-delà des fortifications est accessible par un service de voitures à chevaux (visible à l'arrière-plan) et une ligne de tramway qui la relie à la ville ancienne. La traversée de la Manche, « la plus courte, la plus sûre et la plus agréable » s'effectue en bateau à vapeur (visible à gauche). La ville joue aussi de sa proximité avec Paris avec laquelle elle est reliée en « 3 heures 45 minutes » par « 6 express par jour ». Enfin, le nouvel urbanisme est axé sur la «magnifique digue propre à la construction de villas » sur laquelle donne le nouveau casino, attrait principal de la jeune station, inauguré en 1893.

5. TOULIER, Bernard. L'influence des guides touristiques dans la représentation et la construction de l'espace balnéaire (1850-1950) Dans: Les guides imprimés du XVIe au XXe siècles. Villes, paysages, voyages. Paris : Belin, 2000. P. 239-258.

6. La duchesse de Berry et Dieppe.

7. Kasrener, Jean. Napoléon III à Plombières.

8. SOUVERAINS.

9. Vichy, parc Napoléon III, buste de Napoléon III par Barré.

10. L'enjeu économique des eaux minérales. Energies et matières premières $n^{\circ} 10$. 4ème trimestre 1999.

11. Les autorisations de jeux prévues par l'article $1^{\mathrm{er}}$ de la loi du 15 juin 1907 ne pouvaient être accordées que dans les localités auxquelles le caractère de station hydrominérale et climatique avait été reconnu. Une commission permanente des stations hydrominérales et climatiques est instituée conformément à la loi du 24 septembre 1919. Dix ans plus tard, sont inscrites au tableau des communes érigées en stations hydrominérales et climatiques et dotées d'une chambre 
d'industrie thermale et climatique 25 stations hydrominérales et climatiques, 44 stations hydrominérales et 77 stations climatiques. Voir :

12. Congrès de l'arthritisme à Vittel. Vittel-Journal, $1^{\mathrm{er}}$ mai 1928. n.p.

13. Exemple, avis de l'Académie de médecine du 10 avril 1900 sur la durée de concession des eaux minérales

14. Présentation de la Société française d'hydrologie et de climatologie médicales, succédant à la Société d'hydrologie de Paris, fondée le 21décembre 1853 et reconnue d'utilité publique le 11 juillet 1888,

15. Historique de la thalassothérapie,

16. WEITZ, Georges. Le thermalisme en France au XIXe siècle. Médecine/Sciences, 2002, 18, p. 101-108,

17. Voir par exemple GARDETTE, Victor (dir.). Annuaire médical des stations hydrominérales, climatiques et balnéaires de France (Sanatoriums, Maisons de santé). Paris, 1929. L'ouvrage est subventionné par la Fédération thermale et climatique de France, l'Institut d'hydrologie et de climatologie et l'office national du Tourisme. Il est publié sous le patronage des établissements thermaux de France, avec la collaboration du syndicat général des médecins des stations balnéaires et sanitaires de France et honoré d'une souscription du ministère du Travail, de l'Hygiène et de l'Assistance publique.

18. aquamania.net

19. Mont-Oriol : les eaux troubles,

20. CULOT, Maurice (dir.). La ville d'hiver d'Arcachon, Liège: IFA/Mardaga, $2^{\mathrm{e}}$ édit, 1988. p. 31-54 et

21. Elisée Reclus, d'après JOANNE, Adolphe. Guide de Bordeaux, Arcachon, Royan. Paris : Hachette, 1870

22. [Rôle des artistes et écrivains à Hossegor], $\mathrm{N}^{\circ}$ notice IA00088386.

23. BERTRAND, Nathalie. Tamaris entre Orient et Occident. Arles : Actes Sud, 2003. p. 26-34

24. MARTIN, Maurice. D'Arcachon à Biarritz à travers les Grandes Landes. La Côte d'Argent, partie méridionale, Bordeaux : Imp. G. Gounouilhou, 1907. p. 27

25. Base Mérimée, domaine ArchiXX, Vittel, Grand-Hôtel,

26. Les directeurs d'agences de voyages à Vittel. Vittel-Journal, $1^{\mathrm{er}}$ mai 1928. n.p.

27. Base Mérimée, Hossegor, [Eluere/Hossegor],

28. Base Mérimée, Sainte-Adresse, [Dufayel/Nice-Havrais],

29. Voir par exemple le Groupe Lucien Barrière, anciennement François puis Lucien André qui regroupe encore actuellement 14 casinos et 13 hôtels, Le jeu sauve la mise du Groupe Barrière, Stratégies, avril 2003, $\mathrm{n}^{\circ} 4$,

30. Voir dans ce numéro : Isabelle Barbedor, Les échelles du territoire balnéaire breton. L'exemple de la Côte d'Emeraude.

31. Carte des stations thermales françaises. Sources: Fédération thermale et climatique française,

32. BOYER, Marc. Histoire du tourisme de masse. Paris : PUF, 1999. p. 45.

33. Exemple Chemin de fer du Nord. Excursions à la mer. Saison d'été / G. Fraipont, [Paris]: [Chemins de fer du Nord], [ca 1890] 1 estampe ( affiche ) : lithographie, en coul.; 140 x $100 \mathrm{~cm}$. BNF Richelieu Estampes et photographie Aff. FRAIPONT-Roul. num. 1 .

34. Base ETNOS $\mathrm{N}^{\circ}$ inventaire 993.24.1; A0008 (Numéro de référence)

35. BOUNEAU, Christophe. Réseaux ferroviaires, réseaux électriques et régionalisation en France du milieu du XIXème siècle au second conflit mondial. Contribution à l'histoire économique et sociale des espaces régionaux. Thèse $d$ 'habilitation d'histoire. Paris : Université de Paris IV-Sorbonne, décembre 1998, 3 volumes, 932 p. dactyl. 
36. BOUNEAU, Christophe. La construction et les mutations de l'économie touristique pyrénéenne du milieu du XIXe siècle au second conflit mondial, Actes du congrès de l'association internationale d'Histoire économique, Buenos-Aires, 2002,

37. [Ligne de Cauterets-La Raillère, 1897-1898],

38. Voir dans ce numéro : François Fouger, La place des sociétés de chemin de fer dans la création de la première station alpestre du Revard (Haute-Savoie).

39. Voir dans ce numéro: Paul Smith, La place de l'automobile dans le développement de stations. L'exemple de Deauville.

40. L'histoire de Martigny-les-Bains,

41. Le Touquet (Pas-de-Calais),

42. L'histoire de Cabourg,

43. PITTE, J.-R. Histoire du paysage français de la préhistoire à nos jours. Paris : Taillandier, 2003 (éd. de 1983, revue et augmentée).

44. URBAIN, Jean-Didier. Avenirs de la villégiature balnéaire en France...Téoros, vol. 12, $\mathrm{n}^{\circ} 2$, 1993, p. 40-43,

45. RAMADIER, P. Villégiature et milieux d'affaires. Les lieux de la vie mondaine et de la spéculation foncière. L'exemple de Trouville et Deauville de 1840 à 1900 . Bains de mer et thermalisme en Normandie. XXXVIIe congrès des sociétés historiques et archéologiques de Normandie. Trouville, 18-20 octobre 2001 (à paraître).

46. [Les vacances de Monsieur Hulot],

47. Voir dans ce numéro : François Fray, Le réseau de la clientèle de Barry Dierks.

48. Voir dans ce numéro: Françoise Rollan, Les réseaux d'équipements sportifs dans les stations balnéaires.

49. TOULIER, Bernard. L'assimilation du régionalisme dans l'architecture balnéaire. LOYER, François et TOULIER, Bernard (dir.). Le régionalisme, architecture et identité. Paris : Editions du Patrimoine, coll. Idées et Débats, 2001, p. 96-109

50. [La reconstruction de Royan],

51. Voir dans ce numéro : Gilles Ragot, L'invention du balnéaire « cinquante » à Royan.

52. HOMPS, Hélène. Barcelonnette

53. Voir dans ce numéro: Hélène Homps, Les références culturelles des immigrants "mexicains » à Barcelonnette.

54. VEBLEN, Thorstein. Théorie de la classe de loisir. Paris : Gallimard, 1978, 280 p.(traduit par L. Evrard à partir de The Theory of the Leisure Class. New York : Mac Millan,1899)

55. PICON, Antoine, PRELORENZO, Claude, BORRUEY, René. Territoire, ville et architecture balnéaire. L'exemple de La Grande Motte. Les cahiers de la recherche architecturale, 1993, $3^{\mathrm{e}}$ trim., $n^{\circ} 32 / 33$, p. 59-72,

56. La monoactivité touristique menace de plus en plus le littoral. Le Monde 09/07/2003 


\section{INDEX}

Mots-clés : France, station, villégiature, balnéaire, thermale, climatique, bord de ville, voies de communication, Brighton, Plombières, Aix-les-Bains, duchesse de Berry, Dieppe, Napoléon III, impératrice Eugénie, Saint-Sauveur, Vichy, Bouloumié, Vittel, Pierre Bouloumié, Brosson, ChâtelGuyon, Brocard, le Mont-Dore, La Bourboule, Châteauneuf, Saint-Nectaire, Arcachon, Emile Péreire, Maxime Leroy, Paul Margueritte, Hossegor, George Sand, Tamaris, Edmond Rostand, Cambo-les-Bains, Côte d'Azur, côte landaise, Côte d'Argent, Henry Martinet, Hendaye-Plage, Roger Eluère, René Brouard, Sables-d'Or-les-Pins, Louis Lajarrige, Escoublac-La Baule, Armand Peugeot, Morgat, Ali Pacha, Ernest Dufayel, Sainte-Adresse, François André, Biarritz, Saint-Jeande-Luz, Hendaye, Anglet, Guéthary, Bidart, La Baule, Pornichet, Le Pouliguen, Hyères, Nice, Cannes, Royan, Trouville, Dinard, Le Havre, Dunkerque, Calais, Boulogne, Saint-Malo, côtes de Normandie, côtes de Bretagne, la Manche, Compagnie internationale des wagons-lits, Jean-Raoul Paul, Pierrefitte-Cauterets-Luz, Pierrefitte, Sables-d'Olonne, funiculaires, La Raillère à Cauterets, Tréport, Terrasse, Bagnères-de-Bigorre, Luchon, Barèges, Cauterets, Eaux-Bonnes, Labassere, Gazost, Maurice Martin, Touring Club de France, gare Stella-Plage, Bagnères de Luchon, Antoine Mégret baron d'Etigny, avenue, projet urbain, Charles-E. Isabelle, Martigny-les-Bains, Vernet-lesBains, parc, casino, établissement thermal, villas, grands hôtels, jardin, vacherie, gymnase, villes nouvelles, gare, établissement de bains, stations balnéaires de l'Ouest, cité, Touquet Paris-Plage, Charles Garnier, Thomas Xutler, Mayville, John Robinson Whitley, Le Touquet, Hardelot, StellaPlage, Jaussely

\section{AUTEUR}

\section{BERNARD TOULIER}

Conservateur en chef du patrimoine, Direction de l'architecture et du patrimoine, chercheur associé au CNRS (ex UMR22), responsable du programme « architecture de la villégiature ». bernard.toulier@culture.gouv.fr 